\title{
Assessing the role of undetected colonization and isolation precautions in reducing Methicillin-Resistant Staphylococcus aureus transmission in intensive care units
}

\author{
Theodore Kypraios $^{1 *}$, Philip D O'Neill ${ }^{1}$, Susan S Huang ${ }^{2,3}$, Sheryl L Rifas-Shiman ${ }^{4}$, Ben S Cooper ${ }^{5,6}$
}

\begin{abstract}
Background: Screening and isolation are central components of hospital methicillin-resistant Staphylococcus aureus (MRSA) control policies. Their prevention of patient-to-patient spread depends on minimizing undetected and unisolated MRSA-positive patient days. Estimating these MRSA-positive patient days and the reduction in transmission due to isolation presents a major methodological challenge, but is essential for assessing both the value of existing control policies and the potential benefit of new rapid MRSA detection technologies. Recent methodological developments have made it possible to estimate these quantities using routine surveillance data.

Methods: Colonization data from admission and weekly nares cultures were collected from eight single-bed adult intensive care units (ICUs) over 17 months. Detected MRSA-positive patients were isolated using single rooms and barrier precautions. Data were analyzed using stochastic transmission models and model fitting was performed within a Bayesian framework using a Markov chain Monte Carlo algorithm, imputing unobserved MRSA carriage events.
\end{abstract}

Results: Models estimated the mean percent of colonized-patient-days attributed to undetected carriers as $14.1 \%$ $(95 \% \mathrm{Cl}(11.7,16.5))$ averaged across ICUs. The percent of colonized-patient-days attributed to patients awaiting results averaged $7.8 \%(6.2,9.2)$. Overall, the ratio of estimated transmission rates from unisolated MRSA-positive patients and those under barrier precautions was $1.34(0.45,3.97)$, but varied widely across ICUs.

Conclusions: Screening consistently detected $>80 \%$ of colonized-patient-days. Estimates of the effectiveness of barrier precautions showed considerable uncertainty, but in all units except burns/general surgery and one cardiac surgery ICU, the best estimates were consistent with reductions in transmission associated with barrier precautions.

\section{Background}

Patient isolation measures (such as barrier precautions or physical isolation of patients in single rooms or cohorts) and screening to detect MRSA colonization are core components of almost all policies for preventing the nosocomial transmission of methicillin-resistant Staphylococcus aureus (MRSA). However, there are few well-designed intervention studies considering screening and isolation precautions in the absence of other containment measures, and little is known about the extent to which they reduce transmission. Obtaining estimates

\footnotetext{
* Correspondence: theodore.kypraios@nottingham.ac.uk

${ }^{1}$ School of Mathematical Sciences, University of Nottingham, Nottingham, UK
}

of any such reduction is important for both quantifying the value of existing practices and the potential benefit of new rapid screening technologies.

Screening and isolation measures have two complementary aims: to minimize the number of days MRSApositive patients are left unisolated; and to minimize MRSA transmission from isolated patients. In this paper we consider the extent to which we can quantify the success of control programs in achieving both aims using routine surveillance data. This presents a number of challenges. First, the number of unisolated MRSA patient days usually cannot be directly observed using surveillance data, because in most settings those who 
are known to be MRSA-positive are immediately isolated. Moreover, screening cultures are typically taken no more than weekly. This, combined with less than perfect swab sensitivity, makes it impossible to directly observe the total number of colonized patient days, the number of transmission events and the precise times at which these events occur. Furthermore, estimating the effectiveness of isolation measures in reducing transmission requires quantifying the relative transmission rates of both known and isolated and unobserved and unisolated MRSA positive patients. This cannot be done without estimates of the number of days MRSA-positive patients are left unisolated. Together, these obstacles prevent direct estimates of the efficacy of patient isolation in reducing transmission using routine surveillance data.

Here we show that data augmentation methods allow these problems to be overcome by treating the unobserved times at which patients acquire MRSA as unknown parameters to be estimated. By analysing such surveillance data using stochastic transmission models and making use of data augmentation techniques it is possible to simultaneously estimate the number of undetected MRSA positive patient days and the efficacy of isolation measures in reducing transmission using routinely collected MRSA surveillance data.

\section{Methods}

\section{Data Collection}

We collected detailed census and microbiologic data from eight adult 10-bed intensive care units (ICUs) in a tertiary academic medical center in Boston, Massachusetts where routine admission and weekly bilateral nares screening for MRSA was occurring with high compliance (90\%). Types of ICUs included medical, cardiac, general surgery (2), neurosurgery, thoracic surgery, and cardiac surgery (2). One of the two general surgery wards also received burn and trauma patients. Dates of MRSA-positive clinical cultures (all sources), as well as positive and negative MRSA nares screening cultures were collected during a 17-month period from September 2003 through January 2005. Negative clinical cultures were not assessed. All specimens were processed by routine bacterial culturing techniques. This study was approved by the Institutional Review Board at Brigham and Women's Hospital with a waiver of informed consent.

Newly-identified and previously known MRSA-positive patients were placed under contact precautions consisting of gown and glove use as well as use of single rooms (all ICU rooms were single occupant). Once identified as MRSA-positive, contact precautions were applied on that admission and all subsequent admissions. No nurse cohorting was utilized. Dates of each
ICU admission and discharge were obtained, along with the date on which contact precautions were initially applied (for MRSA or other highly antibiotic resistant pathogens). The first institutional date of a MRSA-positive culture was also recorded, even if it preceded the study period.

\section{Data analysis \\ Stochastic model}

Our baseline analysis used a previously described dynamic stochastic single-ward transmission model to analyse the data [1]. At any point in time, each patient is assumed by this model to be in one of two states, colonized (defined here as the presence of MRSA at any body site, regardless of symptoms) or uncolonized. Each patient entering the ward has a probability $\varphi$ of being already colonized, unless they are already known to be so (i.e. having already had a previous positive test).

During a stay on the ward, an initially uncolonized patient is assumed to have a risk of becoming colonized that can be described by a Poisson process: this means that the probability of a patient becoming colonized between time $t$ and time $t+\tau$ can be approximated by $\lambda$ (t) $\tau$ (where $\tau$ is a small interval of time, the approximation becoming exact as $\tau$ approaches zero). Consequently, a patient's chances of becoming colonized increase with length of stay on the ward. The rate $\lambda(t)$ can vary through time; since we are interested in comparing different potential sources of colonization we assume that $\lambda(t)=\beta_{0}+C(t) \beta_{1}+I(t) \beta_{2}$. Here, $\beta_{0}$ is the rate of background transmission, $\beta_{1}$ is the rate of transmission due to colonized but nonisolated patients, $\beta_{2}$ is the rate of transmission due to colonized and isolated patients, and $C(t)$ and $I(t)$ are the numbers of nonisolated and isolated colonized patients at time $t$, respectively. We thus assume that the "colonization pressure" on an uncolonized individual increases linearly with the number of colonized patients who are isolated and nonisolated. Although it is common practice to use a scaling such as $\beta_{1} / N$ instead of $\beta_{1}$, with $N$ the typical number of patients on the ward, this did not seem necessary since there was very little variation in ward occupancy both between wards and between different times during the study period. The inclusion of the background rate $\beta_{0}$ models the assumption of a constant risk of colonization irrespective of the presence of colonized patients. Such background transmission could, for example, result from environmental contamination or contact with staff carriers of MRSA [2-4]. Note that the model takes no explicit account of $\mathrm{HCW}$ compliance with barrier precautions.

Once a patient is colonized, we assumed that he or she remains so for a three month period. Thus, a patient 
colonized on discharge is assumed to still be colonized if readmitted within three months. This assumption was not critical: the results presented below were virtually unchanged when different periods of colonization were assumed (e.g. 6 months, 9 months). We assumed that the nares culture had a specificity of $100 \%$ and a sensitivity of $p \times 100 \%$, where $p$ (the sensitivity of a nasal swab for detecting MRSA carriage at any site) was estimated from the data.

\section{Statistical inference}

The above model has five main parameters, namely $\varphi, p$, $\beta_{0}, \beta_{1}$, and $\beta_{2}$. We wish to estimate these from the data, which consist of admission times, discharge times, and the times and outcomes of tests. Since our approach involves estimating unseen colonization times, we can also estimate quantities such as the duration of carriage prior to a positive test result. Assessment of the effectiveness of isolation is performed by comparing estimates of $\beta_{1}$ and $\beta_{2}$ : evidence that $\beta_{1}>\beta_{2}$ would support the hypothesis that isolation is effective in reducing transmission. Specifically, we estimate $\mathrm{P}\left(\beta_{1}>\beta_{2}\right)$ and the ratio $\beta_{1} / \beta_{2}$. Estimation was carried out within a Bayesian statistical framework using Markov chain Monte Carlo (MCMC) methods [5].

The five model parameters were assigned uninformative and independent prior distributions: Uniform $(0,1)$ distributions for $\varphi$ and $p$; and exponential distributions with rate $10^{-6}$ for each of the $\beta$ parameters. Since the likelihood of the observed data (dates and outcomes) given the model parameters is computationally intractable, we used a data augmentation method in which the unobserved colonization times were included as additional parameters. This yields an augmented posterior density that is known up to proportionality, which we explored using an MCMC algorithm. Within the algorithm, infection rate parameters were updated using Metropolis-Hastings steps, while both $\varphi$ and $p$ were updated using Gibbs steps [6].

Our methods enable us to estimate the percent of patient days that are attributed to patients who are colonized but not yet detected $\left(p_{\text {hidden }}\right)$, and the percent of patient days attributed to patients who are colonized and have been tested, but who are awaiting test results $\left(p_{\text {wait }}\right)$. Note that $p_{\text {hidden }}>p_{\text {wait }}$ since all "waiting" patients are also "hidden".

Some of the parameter estimates were combined using a random effects model with inverse variance weights [[7], Sec. 5.2] to derive pooled estimates and corresponding standard errors. These computations were carried out using the rmeta package for $\mathrm{R}$ http://www.rproject.org. All other analysis, such as the implementation of the MCMC algorithms, was performed using programs we wrote in $\mathrm{C}$.

\section{Sensitivity analysis}

We refer to the model described above as the full model. To assess the impact of our assumptions, we repeated the analyses using two alternative models. In the first (the no-background model), $\beta_{0}$ is assumed to be zero with high probability (specifically, $\beta_{0}$ was assigned an exponential prior distribution with mean $10^{-4}$ so $\mathrm{P}\left(\beta_{0}\right.$ $<0.001)>0.99$ ). This corresponds to the assumption that virtually all of the MRSA acquisitions result from patient-to-patient transmission (much of which is presumably mediated by transiently colonized healthcare workers, though this is not explicitly modeled). Background transmission unrelated to colonization pressure, e.g. environmental contamination, is assumed to be of negligible importance. In the second (the non-linear model), the assumption of linearly increasing colonization pressure is relaxed, and it is instead assumed that colonization pressure due to both isolated and nonisolated individuals remains constant provided at least one colonized individual is present. Although biologically less plausible than the full model, this model represents an extreme case and by fitting it we obtain insights into the impact of the assumptions underlying the full model. For both the non-linear and no-background models, assessment of the effect of isolation is measured via comparison of the two rates $\beta_{1}$ and $\beta_{2}$ as described above.

\section{Test sensitivity}

We consider two definitions of swab sensitivity: swab sensitivity in detecting colonization of the nares, and swab sensitivity for detecting colonization at any body site. The former is calculated from a subset of the data (without making use of the model) by comparing subsequent swabs in patients who have a first positive swab. Precisely, the sensitivity is estimated by the ratio TP/(TP $+\mathrm{FN}$ ) using serial nares cultures alone, where TP and FN denote total numbers of true positive and false negative tests on a ward excluding the first positive swab from each patient. Since patients, once colonized, are assumed to remain so throughout their ICU stay, TP is simply the number of positive swabs excluding the first for each patient episode, and FN the number of negative swabs that follow an earlier positive for the same patient. The second reported sensitivity is the parameter $p$ estimated from the model making use of cultures from all sites (this is necessarily lower than the sensitivity for detecting nares carriage).

\section{Results}

Descriptive characteristics of the individual ICUs are shown in tables 1 and 2. The median length of stay was two days in all but the two General Surgical wards, in which it was one day. A total of 4,977 MRSA positive 
Table 1 Summary statistics for the study data

\begin{tabular}{lcccccc}
\hline Ward $^{\mathbf{a}}$ & $\begin{array}{c}\text { Number of } \\
\text { patients }\end{array}$ & $\begin{array}{c}\text { Length of stay } \\
\text { Mean(SD) }\end{array}$ & $\begin{array}{c}\text { Percent in contact } \\
\text { precaution }^{\mathbf{b}}\end{array}$ & $\begin{array}{c}\text { Number of swab } \\
\text { tests per person } \\
\text { Mean (SD) }\end{array}$ & $\begin{array}{c}\text { Number of MRSA+ swab } \\
\text { tests per person } \\
\text { Mean (SD) }\end{array}$ & $\begin{array}{c}\text { Number of swab tests taken } \\
\text { after first positive }^{\mathbf{c}}\end{array}$ \\
\hline M1 & 1293 & $3.4(4.7)$ & 11.4 & $1.4(0.8)$ & $0.13(0.5)$ & 73 \\
M2 & 1018 & $4.4(6.4)$ & 19.1 & $1.5(0.9)$ & $0.20(0.61)$ & 45 \\
GS1 & 1227 & $3.4(5.2)$ & 12.4 & $1.3(0.7)$ & $0.14(0.5)$ & 63 \\
GS2 & 1030 & $4.0(8.3)$ & 10.7 & $1.4(0.9)$ & $0.14(0.5)$ & 99 \\
SS1 & 706 & $5.8(11.4)$ & 12.5 & $1.3(0.9)$ & $0.10(0.5)$ & 100 \\
SS2 & 888 & $4.8(9.7)$ & 7.5 & $1.4(1.1)$ & $0.11(0.6)$ & 111 \\
SS3 & 1097 & $3.8(6.4)$ & 6.0 & $1.2(0.7)$ & $0.05(0.3)$ & 51 \\
SS4 & 1263 & $3.6(5.2)$ & 5.1 & $1.4(0.8)$ & $0.07(0.3)$ & 42 \\
\hline
\end{tabular}

a Here M denotes Medical or Cardiac ICU, GS denotes General Surgical ICU, including trauma and burn patients, SS denotes Specialty Surgical ICU.

${ }^{b}$ Includes isolation indicators other than MRSA.

c The total number of swab tests taken after the first MRSA-positive test.

cultures were collected from the study population. Thirty three percent of these cultures were nares screening cultures.

Estimates for $\beta_{1}$ were fairly consistent across the wards while $\beta_{0}$ and $\beta_{2}$ exhibited more variation (figure 1 ). General surgery wards were distinguished by the fact that the estimated transmission rate with barrier precautions $\left(\beta_{2}\right)$ was found to be higher than that without $\left(\beta_{1}\right)$. Posterior distributions of the three rates were found to be negatively correlated. This was expected since an increase in one rate must be accounted for by a decrease in another. The background rate $\beta_{0}$ had considerable correlation with the other two rates (range -0.23 to -0.58 ), while $\beta_{1}$ and $\beta_{2}$ were far less strongly correlated (range -0.05 to -0.30 ).

\section{The impact of undetected colonized patients on} transmission

Approximately $15 \%$ of colonized patient days (CPDs) were due to undetected patients, this being made up of around $10 \%$ due to patients awaiting test results, and about 5\% due to patients who had not been tested (table
3). The fraction of CPD attributed to undetected patients was not statistically associated with the mean monthly overall prevalence or admission prevalence of MRSA in each ICU (Pearson's correlation coefficient -0.58 and -0.53 , p-values $0.13,0.17$, respectively.)

\section{Is isolation effective? Comparison of $\beta_{1}$ and $\beta_{2}$}

In five of the eight wards there was consistent, though weak, evidence that $\beta_{1}$ is larger than $\beta_{2}$ indicating that isolation is associated with reduced transmissibility (table 4). The probability of an isolation benefit varied widely across the units, e.g. $27-82 \%$ under the full model.

\section{Pooled estimate of the effectiveness of isolation}

Although our analysis treats each ward individually, it is nevertheless of interest to consider a pooled estimate of isolation efficacy. Such an estimate of $\left(\beta_{1} / \beta_{2}\right)$ across all wards has mean ( $95 \%$ credible interval) $1.34(0.45$, 3.97) which indicates some evidence to support the efficacy of isolation, but a relatively high degree of uncertainty. This estimate can be decomposed into

Table 2 Observed prevalence and incidence for the study data

\begin{tabular}{cccc}
\hline Ward $^{\mathbf{a}}$ & $\begin{array}{c}\text { Monthly MRSA } \\
\text { prevalence }^{\mathbf{b}} \\
\text { Mean (SD) }\end{array}$ & $\begin{array}{c}\text { Monthly MRSA } \\
\text { admission prevalence } \\
\text { Mean (SD) }\end{array}$ & $\begin{array}{c}\text { Monthly MRSA incidence density (new cases per 1,000 patient days) } \\
\text { Mean (SD) }\end{array}$ \\
\hline M1 & $16.9(4.0)$ & $12.6(3.0)$ & $8.8(8.1)$ \\
M2 & $23.5(4.7)$ & $20.6(3.3)$ & $5.8(5.8)$ \\
GS1 & $20.5(5.8)$ & $15.4(4.2)$ & $9.3(8.3)$ \\
GS2 & $19.5(6.4)$ & $10.9(4.1)$ & $18.2(9.7)$ \\
SS1 & $21.1(8.7)$ & $12.9(7.5)$ & $9.8(10.1)$ \\
SS2 & $13.8(4.4)$ & $7.3(2.8)$ & $6.8(4.9)$ \\
SS3 & $10.7(3.9)$ & $6.3(3.6)$ & $9.4(7.9)$ \\
SS4 & $9.2(3.3)$ & $4.5(3.1)$ & $9.1(6.1)$ \\
\hline
\end{tabular}

${ }^{a}$ Here M denotes Medical or Cardiac ICU, GS denotes General Surgical ICU, including trauma and burn patients, SS denotes Specialty Surgical ICU.

${ }^{b}$ The number of ICU patients ever known to be MRSA-positive before or during that month, divided by the total number of ICU patients that month.

c The number of patients ever known to be MRSA positive before or within two calendar days of admission, divided by the total number of monthly admissions. 


\section{Marginal posterior distributions of the colonization rates for each ward.}

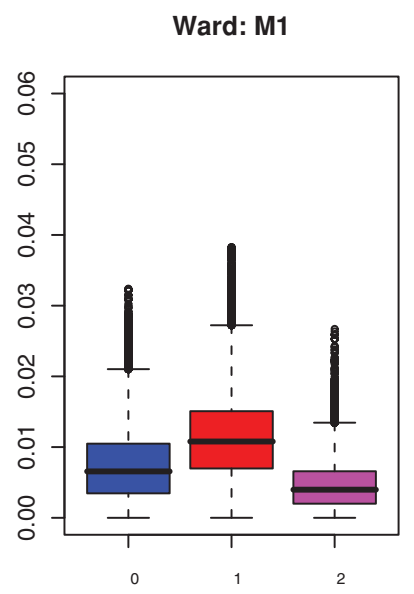

Ward: M2

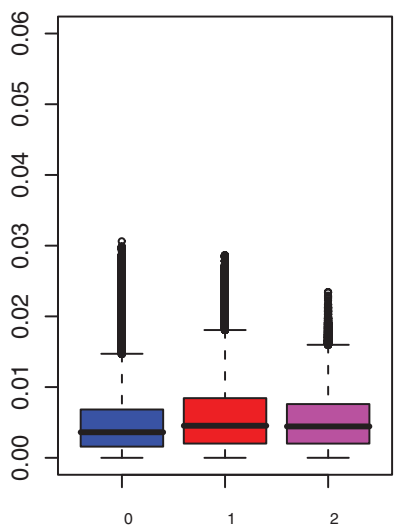

Ward: SS1

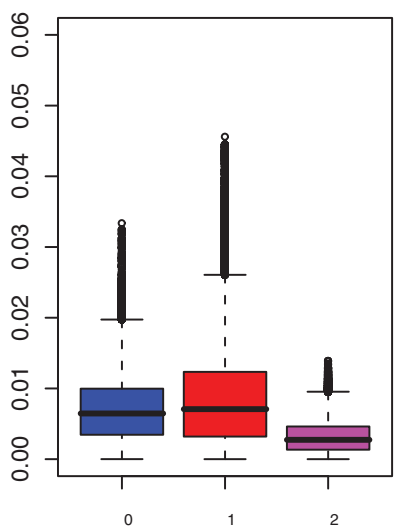

Ward: SS4

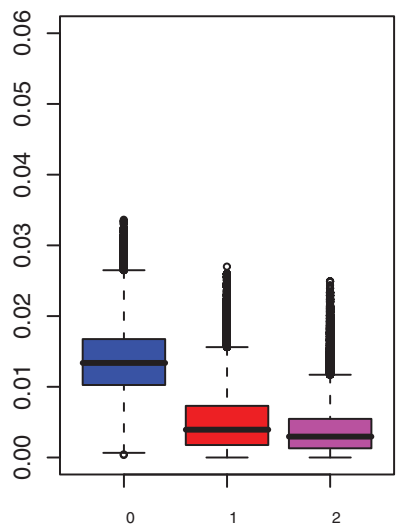

Ward: GS1

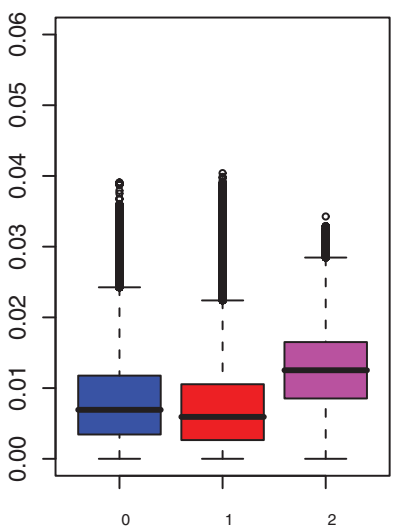

Ward: SS2

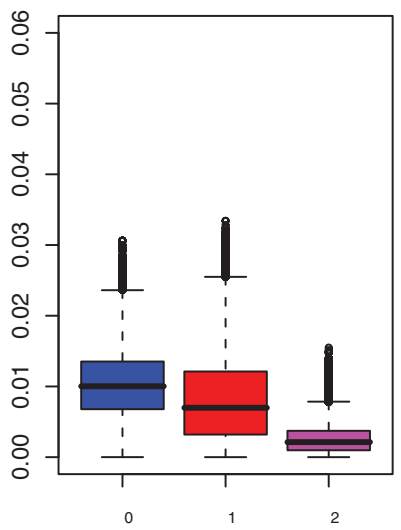

All Wards

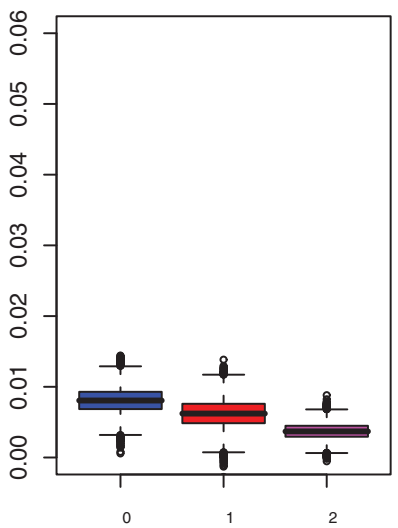

Figure 1 Marginal posterior distributions of the colonization rates for each ward. Boxplots of the marginal posterior distributions are shown which contain (the smallest observation, lower quartile (Q1), median (Q2), upper quartile (Q3), and largest observation (sample maximum) as well as any outliers. The whiskers extend from the edges of the box to the most extreme data point which is no more than 1.5 times the interquartile range away from the box. 
Table 3 Assessment of undetected colonization

\begin{tabular}{|c|c|c|c|c|c|c|}
\hline \multirow[t]{2}{*}{ Ward } & \multicolumn{2}{|c|}{ Full model } & \multicolumn{2}{|c|}{ No-background } & \multicolumn{2}{|c|}{ Non-linear } \\
\hline & $p_{\text {hidden }}$ & $p_{\text {wait }}$ & $p_{\text {hidden }}$ & $p_{\text {wait }}$ & $p_{\text {hidden }}$ & $p_{\text {wait }}$ \\
\hline M1 & $\begin{array}{l}16.5 \\
(14.9 \\
18.2)\end{array}$ & $\begin{array}{l}11.5 \\
(10.6, \\
12.4)\end{array}$ & $\begin{array}{l}16.6 \\
(14.9, \\
18.2)\end{array}$ & $\begin{array}{c}11.4 \\
(10.5, \\
12.2)\end{array}$ & $\begin{array}{c}16.4 \\
(14.8, \\
18.1)\end{array}$ & $\begin{array}{c}11.5 \% \\
(10.6, \\
12.3)\end{array}$ \\
\hline M2 & $\begin{array}{c}10.5 \\
(8.3,13.2)\end{array}$ & $\begin{array}{c}6.7 \\
(5.6,7.9)\end{array}$ & $\begin{array}{c}10.8 \\
(8.4,13.7)\end{array}$ & $\begin{array}{c}6.9 \\
(5.8,8.3)\end{array}$ & $\begin{array}{c}10.4 \\
(8.1,13.3)\end{array}$ & $\begin{array}{c}6.7 \\
(5.5,8.0)\end{array}$ \\
\hline GS1 & $\begin{array}{c}13.8 \\
(12.2 \\
15.5)\end{array}$ & $\begin{array}{c}8.3 \\
(7.6,8.9)\end{array}$ & $\begin{array}{c}13.9 \\
(12.3 \\
15.6)\end{array}$ & $\begin{array}{c}8.3 \\
(7.6,8.9)\end{array}$ & $\begin{array}{c}13.9 \\
(12.2 \\
15.5)\end{array}$ & $\begin{array}{c}8.3 \\
(7.5,8.9)\end{array}$ \\
\hline GS2 & $\begin{array}{c}17.3 \\
(15.1 \\
19.8)\end{array}$ & $\begin{array}{c}7.4 \\
(6.6,8.2)\end{array}$ & $\begin{array}{c}17.3 \\
(15.1 \\
19.6)\end{array}$ & $\begin{array}{c}7.3 \\
(6.6,8.0)\end{array}$ & $\begin{array}{c}17.4 \\
(15.1 \\
19.7)\end{array}$ & $\begin{array}{c}7.5 \\
(6.7,8.2)\end{array}$ \\
\hline SS1 & $\begin{array}{c}9.6 \\
(8.1,11.2)\end{array}$ & $\begin{array}{c}4.7 \\
(4.4,4.9)\end{array}$ & $\begin{array}{c}9.9 \\
(8.4,11.6)\end{array}$ & $\begin{array}{c}4.7 \\
(4.4,4.9)\end{array}$ & $\begin{array}{c}9.6 \\
(8.1,11.2)\end{array}$ & $\begin{array}{c}4.7 \\
(4.4,4.9)\end{array}$ \\
\hline SS2 & $\begin{array}{c}10.7 \\
(9.0,12.7)\end{array}$ & $\begin{array}{c}5.7 \\
(5.4,6.1)\end{array}$ & $\begin{array}{c}10.9 \\
(9.1,13.0)\end{array}$ & $\begin{array}{c}5.8 \\
(5.4,6.1)\end{array}$ & $\begin{array}{c}10.7 \\
(8.9,12.8)\end{array}$ & $\begin{array}{c}5.7 \\
(5.4,6.1)\end{array}$ \\
\hline SS3 & $\begin{array}{l}15.6 \\
(13.0 \\
18.4)\end{array}$ & $\begin{array}{c}7.9 \\
(6.9,8.7)\end{array}$ & $\begin{array}{r}16.2 \\
(13.5, \\
18.9)\end{array}$ & $\begin{array}{c}7.8 \\
(6.9,8.6)\end{array}$ & $\begin{array}{l}15.5 \\
(12.7 \\
18.3)\end{array}$ & $\begin{array}{c}7.9 \\
(6.9,8.7)\end{array}$ \\
\hline SS4 & $\begin{array}{l}19.8 \\
(16.2, \\
23.3)\end{array}$ & $\begin{array}{c}10.1 \\
(8.7,11.4)\end{array}$ & $\begin{array}{l}20.7 \\
(17.1 \\
24.3)\end{array}$ & $\begin{array}{c}9.7 \\
(8.4,10.9)\end{array}$ & $\begin{array}{l}19.8 \\
(16.5 \\
23.3)\end{array}$ & $\begin{array}{c}10.1 \\
(8.6,11.3)\end{array}$ \\
\hline
\end{tabular}

Note: Model estimates (95\% credible intervals) of the mean percent of colonized-patient-days attributed to undetected colonized patients $\left(p_{\text {hidden }}\right)$, and the mean percent of colonized-patient-days attributed to colonized patients who had been swabbed but were waiting for results $\left(p_{\text {wait }}\right)$.

contributions from medical, specialty surgical and general surgical wards, yielding 1.89 (0.21 16.60), 1.73 $(0.338 .94)$ and $0.7(0.1,5.0)$ respectively. Variation across all wards is illustrated in Figure 2, which shows a pooled estimate of $\log \left(\beta_{1} / \beta_{2}\right)$ for the full modeland the corresponding standard deviation for each ward. In addition, the value of the $\mathrm{I}^{2}$ statistic, which describes the percentage of variation across wards that is due to heterogeneity, was equal to zero for the aforementioned pooled estimates.

Table 4 Assessment of isolation efficacy

\begin{tabular}{lcccccc}
\hline Ward & \multicolumn{2}{c}{ Full model } & \multicolumn{2}{c}{ No-background } & \multicolumn{2}{c}{ Non-linear } \\
\cline { 2 - 7 } & $\mathrm{P}\left(\boldsymbol{\beta}_{\mathbf{1}}>\boldsymbol{\beta}_{\mathbf{2}}\right)$ & $\begin{array}{c}\text { Median } \\
\left(\boldsymbol{\beta}_{\mathbf{1}} / \boldsymbol{\beta}_{\mathbf{2}}\right)\end{array}$ & $\mathrm{P}\left(\boldsymbol{\beta}_{\mathbf{1}}>\boldsymbol{\beta}_{\mathbf{2}}\right)$ & $\begin{array}{c}\text { Median } \\
\left(\boldsymbol{\beta}_{\mathbf{1}} / \boldsymbol{\beta}_{\mathbf{2}}\right)\end{array}$ & $\mathrm{P}\left(\boldsymbol{\beta}_{\mathbf{1}}>\boldsymbol{\beta}_{\mathbf{2}}\right)$ & $\begin{array}{c}\text { Median } \\
\left(\boldsymbol{\beta}_{\mathbf{1}} / \boldsymbol{\beta}_{\mathbf{2}}\right)\end{array}$ \\
\hline M1 & 0.82 & 2.7 & 0.83 & 2.4 & 0.75 & 2.3 \\
M2 & 0.51 & 1.0 & 0.54 & 1.1 & 0.45 & 0.8 \\
GS1 & 0.27 & 0.5 & 0.15 & 0.3 & 0.36 & 0.6 \\
GS2 & 0.50 & 1.0 & 0.58 & 1.2 & 0.62 & 1.5 \\
SS1 & 0.73 & 2.7 & 0.57 & 1.3 & 0.53 & 1.1 \\
SS2 & 0.79 & 3.3 & 0.79 & 2.0 & 0.71 & 2.0 \\
SS3 & 0.44 & 0.8 & 0.60 & 1.3 & 0.67 & 2.0 \\
SS4 & 0.58 & 1.3 & 0.70 & 2.3 & 0.59 & 1.4 \\
\hline
\end{tabular}

Note. $\mathrm{P}\left(\beta_{1}>\beta_{2}\right)$, the probability that $\beta_{1}$ is larger than $\beta_{2}$, measures the strength of the evidence that isolation was associated with reduced transmission (a value of 1 would indicate certainty), and the ratio of $\beta_{1}$ to $\beta_{2}$ measures the relative infectivity of unisolated compared to unisolated patients.

\section{Test sensitivity}

We report the two estimates of swab sensitivity defined in the methods section (table 5). Overall, the sensitivity of detecting colonization at any body site was estimated from the model to be $60 \%$ (95\% 55\%-63\%) while the sensitivity of the nares test was estimated directly from the data to be $85 \%$.

\section{Percentage of new admissions already colonized}

Each patient admitted to a ward during the study period may be known to be colonized due to a previous positive test. If not, colonization status is unknown, since the patient may be either new to the study, or else previously admitted but with no positive test result. The parameter $\varphi$ in our model represents the proportion of such patients who are colonized on admission. Note that $\varphi$ should not be interpreted as a measure of admission prevalence, since it excludes known positives.

Estimates of $\varphi$ were found to be similar to the percentage of patients admitted with a known previous positive test on all wards except M2, with the lowest values tending to occur in the specialist surgery wards (table 6). Estimates from the full model and no-background model were similar, as were estimates from the non-linear model (not shown).

\section{Discussion}

We have shown that a data-augmentation approach allows us to estimate the extent of undetected MRSA carriage and the effectiveness of barrier precautions (gown and gloves) in preventing transmission using only routinely collected high-compliance admission and weekly MRSA screening cultures. Such estimates would have been difficult or impossible with more conventional approaches and would have required assuming rather than estimating the unobserved carriage data.

Our results have shown that admission and weekly nares screening with conventional culture methods are likely to have detected at least $80 \%$ of MRSA colonized patient days in the ICUs studied, despite 48-hour culture turn-around times, and sensitivities to detect MRSA carriage of only $85 \%$ for detecting nares carriage and $59 \%$ for carriage at any body site. The explanation for this high detection of MRSA patient days despite a low sensitivity for non-nares sites is that clinical cultures of other sites are routinely being performed for medical reasons. Thus, nares screening in conjunction with usual routine culturing performed for medical reasons captures a large majority of positive MRSA carriers. While generalizability to non-ICU wards remains unclear, this level of detection in ICUs is reassuring since risk of MRSA infection is highest in these settings.

We estimate that approximately $10 \%$ of potential contact precaution days are missed due to delays in 


\section{Effectiveness of isolation precautions}

M1

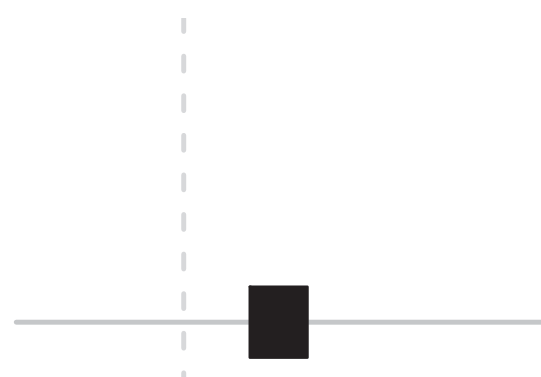

M2

GS1

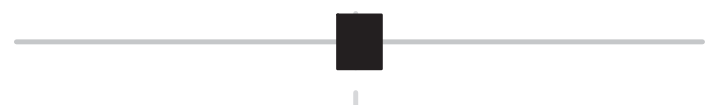

GS2

SS1

$\frac{\text { ㅁํำ }}{3}$

SS2

SS3

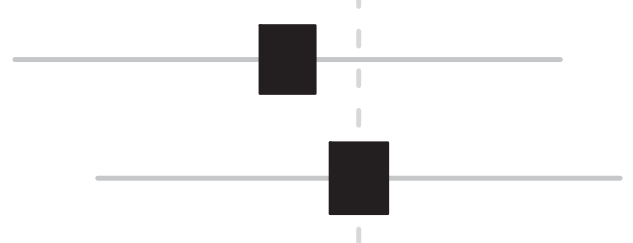

SS4

Summary
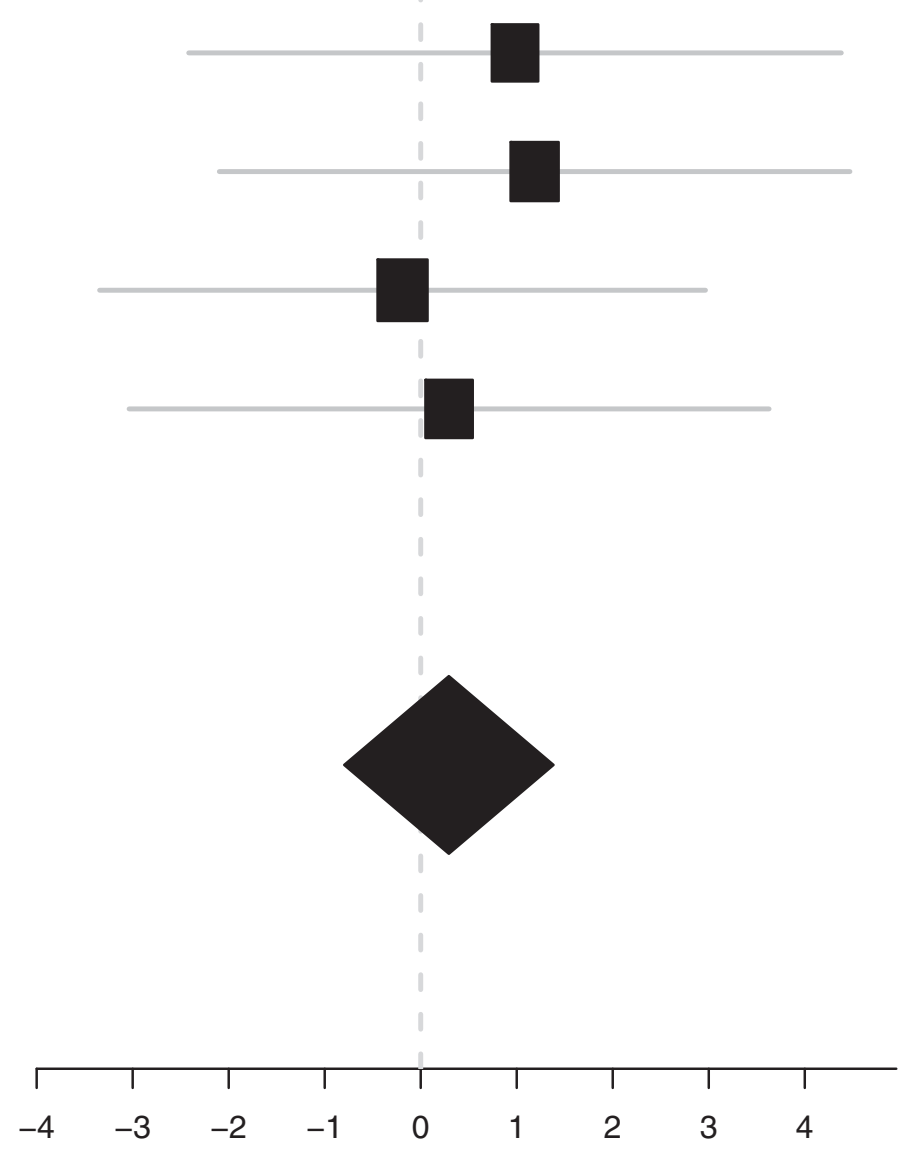

\section{Effect}

Figure 2 Effectiveness of isolation precautions. Forest plot showing individual and pooled estimate of $\ln \left(\beta_{1} / \beta_{2}\right)$ for each ward. Horizontal lines are $95 \% \mathrm{Cls}$ and the size of each square is proportional to weight in the meta-analysis. The end points of the summary diamond indicate $95 \% \mathrm{Cl}$. 
Table 5 Two measures of nares test sensitivity based upon serial nares cultures

\begin{tabular}{|c|c|c|}
\hline Ward & $\begin{array}{l}\text { Sensitivity of Detecting } \\
\text { Nares Colonization }(\%)^{\mathrm{a}}\end{array}$ & $\begin{array}{l}\text { Sensitivity of detecting } \\
\text { colonization at any body site } \\
(\%): \text { Median }(95 \% \mathrm{Cl})^{\mathrm{b}}\end{array}$ \\
\hline M1 & 92 & $64(56,71)$ \\
\hline M2 & 84 & $56(47,65)$ \\
\hline GS1 & 82 & $61(54,69)$ \\
\hline GS2 & 88 & $50(42,57)$ \\
\hline SS1 & 89 & $62(54,68)$ \\
\hline SS2 & 89 & $68(60,76)$ \\
\hline SS3 & 85 & $55(45,64)$ \\
\hline SS4 & 72 & $54(44,65)$ \\
\hline Overall & 85 & $59(55,63)$ \\
\hline
\end{tabular}

${ }^{a}$ Given by the ratio TP/(TP+FN) where TP is the number of positive swabs excluding the first for each patient episode and FN the number of negative swabs that follow an earlier positive for the same patient.

${ }^{b}$ The parameter $p$ estimated from the model making use of cultures from all sites.

obtaining test results. If contact precautions cause a $28.1 \%$ reduction in the transmission rate (i.e. $1-\left(\beta_{2} / \beta_{1}\right)$, using the pooled estimate from all the wards), then an additional $5 \%$ reduction in total transmission rates would be gained with instantaneous swab results, suggesting a limit to the potential benefit in using rapid screening technologies. However, the potential benefit of rapid test results would increase in line with the effectiveness of isolation measures.

While our best estimate is that gown and glove barrier precautions produce a $28 \%$ reduction in MRSA transmission, we recognize that these estimates have considerable uncertainty despite including over 11 ward-years of data and there is a substantial chance that the effect is both much lower and much larger. Thus we estimate that there is $30 \%$ chance that the reduction in transmission is above $44 \%$ and a $30 \%$ chance that isolation actually increases transmission.
This uncertainty may indicate that many more years of data are required, and/or may be explained by the fact that data from different types of ICUs were pooled. However the Woolf's test for heterogeneity did not reach significance at a $5 \%$ level $(\mathrm{p}=0.982)$, indicating that these differences are consistent with chance. Moreover since comparisons between ward-types were not planned they may be of value for generating, but not testing hypotheses.

Recognizing these caveats, it is plausible that barrier precautions have differential effectiveness in different types of ICUs. The estimates found in this study suggest that medical ICUs may derive the most benefit from barrier precautions. This is important since the MRSA prevalence in medical ICUs is often higher than in other ICU types. Interestingly, our results suggest that no benefit was derived in general surgery ICUs serving burn and trauma patients. It is possible that barrier precautions may be less effective when the burden of MRSA is profuse, such as with surgical wounds or burns. Such an interpretation is supported by the fact that the rate of background transmission $\left(\beta_{0}\right)$ is substantially higher on these units. This is also supported by other work in these units suggesting that contamination with MRSA is higher in surgical versus medical ICUs [8]. Another possible explanation for the lack of benefit seen in select wards is low compliance with contact precautions, although this was not assessed. Finally, if barrier precautions provide a false sense of security and lead to poorer hand hygiene, then transmission might effectively worsen with this practice.

It is also possible that the differing effectiveness of barrier precautions among ICUs could be due to unmeasured effects. Specifically, we did not measure or account for variations in host risk factors for acquisition (comorbidities, severity of illness, wounds, devices), nor did we measure the effect of other ongoing infection control interventions such as hand hygiene campaigns.

Table 6 Percentage of patients who are colonized on admission to a ward

\begin{tabular}{cccc}
\hline Ward No Percent having a previous positive test ${ }^{\mathbf{a}}$ & \multicolumn{2}{c}{$\begin{array}{c}\text { Estimated percent of MRSA carriers among admissions with unknown status } \mathbf{b}(\boldsymbol{\varphi} \times \\
\mathbf{1 0 0 \%}) \mathbf{M e d i a n}(\mathbf{9 5 \%} \mathbf{C l})\end{array}$} \\
\cline { 2 - 4 } & & Full Model & No Background \\
\hline M1 & 8.4 & $9.4(7.3,12)$ & $9.6(7.5,12.2)$ \\
M2 & 7.7 & $14.1(10.3,18.6)$ & $14.4(10.7,19)$ \\
GS1 & 8.1 & $10.4(7.8,13.4)$ & $10.7(8.1,13.9)$ \\
GS2 & 5.7 & $8.3(5.4,12.1)$ & $9.0(6,13)$ \\
SS1 & 8.2 & $8.7(5.9,12.2)$ & $9.2(6.4,12.7)$ \\
SS2 & 4.6 & $5.7(3.8,8.2)$ & $6.1(4.2,8.6)$ \\
SS3 & 4.2 & $3.4(2.1,5.3)$ & $3.8(2.4,5.8)$ \\
SS4 & 3.5 & $4.6(2.9,6.9)$ & $5.9(4.0,8.4)$ \\
\hline
\end{tabular}

a Patients known to be colonized due to having had a previous positive test during the study period (column 2).

${ }^{b}$ The percentage of patients with unknown status (due to being newly-admitted or with no previous positive test) who are estimated to be colonized on admission by the model. 
We do note that infection control interventions were not differentially applied to certain ICUs during this time, but levels of compliance are not known. In the absence of reliable data about these factors, none were considered in our analysis.

Our results may underestimate the full effect of barrier precautions since all ICU rooms were single occupancy; thus, we could not assess the impact of this component. In addition, studies have suggested that compliance with barrier precautions is around 70\% $[9,10]$. Our estimates represent estimates of "effectiveness" (under routine operation) rather than a theoretical efficacy that would apply only under perfect compliance. Thus, while our estimates can be considered conservative, we believe they are the most practically relevant outcome measures. More precise estimates would require more prolonged surveillance data, data with more frequent swabbing intervals, and/or extensive typing information to help identify transmission routes.

The model-based approach that we have adopted for data analysis has several advantages over standard statistical methods, yielding more powerful analyses based on more realistic assumptions. In particular, it accounts for unobserved but important events (e.g. the time at which a patient becomes colonized), and attempts to estimate these events and their uncertainty. It also is based upon biologically meaningful assumptions regarding transmission, addressing both environmental sources and patient-to-patient transfer.

In summary, we have used a stochastic model to estimate the importance of undetected MRSA carriage and the impact of gown and glove precautions on MRSA transmission from 11-ICU years of active MRSA surveillance data. Such isolation precautions are the foundation of infection control guidelines for MRSA control. Nevertheless, there are concerns that isolation techniques may reduce the quality of patient care and incur risks related to inattention [11-13]. Thus, quantifying the beneficial effects and understanding the types of hospital wards in which such measures are likely to be insufficient is a critical part of weighing the need for additional or alternative measures. This work shows that even with conventional MRSA detection technology and weekly screening, a very large proportion of the total patient MRSA reservoir can be detected. It also suggests that barrier precautions may afford substantial benefit in medical and possibly specialty surgical ICUs, but that barriers to its effectiveness may need further study. The large uncertainty in effectiveness estimates, however, illustrate the limitations of routine surveillance data and highlight the need for well-designed prospective intervention studies to evaluate such interventions with greater precision.

\section{Conclusions}

In a study of 8 ICUs performing admission and weekly nares surveillance, the average percentage of colonizedpatient-days attributed to undetected carriers was $14 \%$, while the percentage of colonized-patient-days attributed to patients awaiting test results was $8 \%$. This suggests that nares surveillance identifies a large majority of carriers and that pcr testing may confer only a small benefit over routine culture. Estimates of the effectiveness of barrier precautions showed an overall benefit of $25 \%$, but this benefit varied widely across different types of ICUs.

\section{Acknowledgements}

TK would like to acknowledge funding from Wellcome Trust (Ref: 076850). Data collection was funded by the CDC Prevention Epicenters Program and the National Institutes of Health (K23Al64161, Huang).

\section{Author details}

${ }^{1}$ School of Mathematical Sciences, University of Nottingham, Nottingham, UK. ${ }^{2}$ Division of Infectious Diseases and Health Policy Research Institute, University of California Irvine School of Medicine, Irvine, California, USA. ${ }^{3}$ Channing Laboratory, Brigham and Women's Hospital and Harvard Medical School, Boston, Massachusetts, USA. ${ }^{4}$ Department of Ambulatory Care and Prevention, Harvard Medical School and Harvard Pilgrim Health Care, Boston, Massachusetts, USA. ${ }^{5}$ Centre for Clinical Vaccinology and Tropical Medicine, Nuffield Department of Clinical Medicine, University of Oxford, Oxford, UK. ${ }^{6}$ Faculty of Tropical Medicine, Mahidol University, Bangkok, Thailand.

\section{Authors' contributions}

TK coded and implemented the statistical analysis. BSC and PDO conceived the modeling and statistical framework, and drafted the manuscript. SSH contributed content expertise, oversaw dataset preparation for modeling, and helped draft the manuscript. SSR-S contributed to the data cleaning and preparation of the dataset for use in the models. All authors have read and approved the final manuscript.

\section{Competing interests}

The authors declare that they have no competing interests.

\section{Received: 19 May 2009}

Accepted: 16 February 2010 Published: 16 February 2010

\section{References}

1. Forrester ML, Pettitt AN, Gibson GJ: Bayesian inference of hospitalacquired infectious diseases and control measures given imperfect surveillance data. Biostatistics 2007, 8(2):383-401.

2. Snyder GM: Detection of methicillin-resistant Staphylococcus aureus and vancomycin-resistant enterococci on the gowns and gloves of healthcare workers. Infect Control Hosp Epidemiol 2008, 29(7):583-589.

3. Huang SS, Datta R, Platt R: Risk of acquiring antibiotic-resistant bacteria from prior room occupants. Arch Intern Med 2006, 166:1945-1951.

4. Boyce JM, Havill NL, Otter JA, Adams NM: Widespread environmental contamination associated with patients with diarrhea and methicillinresistant Staphylococcus aureus colonization of the gastrointestinal tract. Infect Control Hosp Epidemiol 2007, 28:1142-1147.

5. O'Neill PD: A tutorial introduction to Bayesian inference for stochastic epidemic models using Markov chain Monte Carlo methods. Mathematical Biosciences 2002, 180:103-114.

6. Gilks WR, Richardson S, Spiegelhalter DJ, Eds: Markov chain Monte Carlo in practice London: Chapman and Hall 1996.

7. Sutton AJ, Abrams KR, Jones DR, Sheldon TA, Song F: Methods for metaanalysis in medical research Chichester: Wiley 2000.

8. Goodman ER, Platt R, Bass R, Onderdonk AB, Yokoe DS, Huang SS: Impact of an environmental cleaning intervention on cultures for MRSA and VRE. Infect Control and Hosp Epidemiol 2008, 29(7):593-599. 
9. Weber DJ, Sickbert-Bennett EE, Brown VM, Brooks RH, Kittrell IP, Featherstone BJ, Adams TL, Rutala WA: Compliance with isolation precautions at a university hospital. Infect Control Hosp Epidemiol 2007, 28(3):358-361.

10. Manian FA, Ponzillo JJ: Compliance with routine use of gowns by healthcare workers ( $\mathrm{HCWs}$ ) and non-HCW visitors on entry into the rooms of patients under contact precautions. Infect Control Hosp Epidemiol 2007, 28(3):337-340.

11. Kirkland KB, Weinstein JM: Adverse effects of isolation. Lancet 1999, 354:1177-1178.

12. Saint $S$, Higgins LA, Nallamothu BK, Chenoweth C: Do physicians examine patients in contact isolation less frequently? A brief report. Am J Infect Control 2003, 31:354-356.

13. Catalano G, Houston SH, Catalano MC, Butera AS, Jennings SM, Hakala SM, Burrows SL, Hickey MG, Duss CV, Skelton DN, Laliotis GJ: Anxiety and depression in hospitalized patients in resistant organism isolation. South Med J 2003, 96:141-145.

\section{Pre-publication history}

The pre-publication history for this paper can be accessed here:http://www. biomedcentral.com/1471-2334/10/29/prepub

doi:10.1186/1471-2334-10-29

Cite this article as: Kypraios et al: Assessing the role of undetected colonization and isolation precautions in reducing

Methicillin-Resistant Staphylococcus aureus transmission in intensive care units. BMC Infectious Diseases 2010 10:29.

\section{Submit your next manuscript to BioMed Central and take full advantage of:}

- Convenient online submission

- Thorough peer review

- No space constraints or color figure charges

- Immediate publication on acceptance

- Inclusion in PubMed, CAS, Scopus and Google Scholar

- Research which is freely available for redistribution

Submit your manuscript at www.biomedcentral.com/submit 